IRA-International Journal of Applied Sciences ISSN 2455-4499; Vol.05, Issue 02 (2016)

Institute of Research Advances

Pg. no. 85-90

http://research-advances.org/index.php/IRAJAS

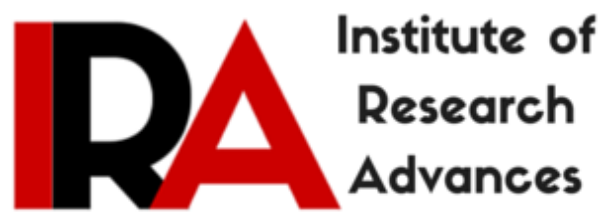

\title{
An Overview of Hyperspectral Remote Sensing and its applications in various Disciplines
}

\author{
${ }^{1}$ Alpana Shukla, ${ }^{2}$ Rajsi Kot \\ 1,2 Department of Botany, \\ M. G. Science Institute, India.
}

Type of Review: Peer Reviewed.

DOI: http://dx.doi.org/10.21013/jas.v5.n2.p4

\section{How to cite this paper:}

Shukla, A., \& Kot, R. (2016). An Overview of Hyperspectral Remote Sensing and its applications in various Disciplines. IRA-International Journal of Applied Sciences (ISSN 2455-4499), 5(2), 85-90. doi:http://dx.doi.org/10.21013/jas.v5.n2.p4

(C) Institute of Research Advances

\section{(cc) EY-NO}

This work is licensed under a Creative Commons Attribution-Non Commercial 4.0 International License subject to proper citation to the publication source of the work.

Disclaimer: The scholarly papers as reviewed and published by the Institute of Research Advances (IRA) are the views and opinions of their respective authors and are not the views or opinions of the IRA. The IRA disclaims of any harm or loss caused due to the published content to any party. 


\section{ABSTRACT}

Recent advances in remote sensing and geographic information has opened new directions for the development of hyperspectral sensors. Hyperspectral remote sensing, also known as imaging spectroscopy is a new technology. Hyperspectral imaging is currently being investigated by researchers and scientists for the detection and identification of vegetation, minerals, different objects and background. Hyperspectral remote sensing combines imaging and spectroscopy in a single system which often includes large data sets and requires new processing methods. Hyperspectral data sets are generally made of about 100 to 200 spectral bands of relatively narrow bandwidths (5-10 nm), whereas, multispectral data sets are usually composed of about 5 to 10 bands of relatively large bandwidths (70$400 \mathrm{~nm}$ ). Hyperspectral imagery is collected as a data cube with spatial information collected in the X-Y plane, and spectral information represented in the Z-direction. Hyperspectral remote sensing is applicable in many different disciplines. It was originally developed for mining and geology; it has now spread into fields such as agriculture and forestry, ecology, coastal zone management, geology and mineral exploration. This paper presents an overview of hyperspectral imaging, data exploration and analysis, applications in various disciplines, advantages and disadvantages and future aspects of the technique.

Keywords: Hyperspectral remote sensing, Spectroscopy, multispectral data, geology, mineral exploration.

\section{INTRODUCTION}

In earth observation, applicability of multi-spectral data to improve geometric resolution of sensors is being investigated by researchers and scientists. It is important to acquire accurate quantitative information of land surface parameters to understand the bio-geophysical processes in terrestrial. Therefore, quantification of many parameters as well as inventory of different type based upon spectral response in various wavelength regions is very crucial. Hyperspectral remote sensing from airborne and satellite systems have been utilized as a data source for numerous remote sensing applications over the past two decades. Hyperspectral imaging is widely accepted technology and quickly moving into the mainstream of remote sensing research studies [2]. Here, hyperspectral means spectra having large number of narrow, contiguously spaced spectral bands. The term hyperspectral is also known as spectroscopy, spectrometry and spectroradiometry [3]. Spectroscopy is a branch of physics concerned with the production, transmission, measurement and interpretation of electromagnetic spectra [7]. Spectrometry or spectroradiometry is derived from spectro-photometry, the measure of photons as a function of wavelength. Reflectance spectra of natural surface are acquired by Spectrometers used in laboratories, field, aircraft or satellites [8]. When an image is constructed from an imaging spectrometer that records the spectra for contiguous image pixels, it is referred as imaging spectroscopy, imaging spectrometry or hyperspectral imaging [8]. Hyperspectral imaging is a new technique for obtaining a spectrum in each position of a large array of spatial positions so that any one spectral wavelength can be used to make a recognisable image [3]. Due to specific chemical bonds in materials and the spectral features in each pixel it is possible to investigate and map materials spatially [3]. The narrow spectral channels are able to detect minor spectral features that might otherwise be undetected within the broader bands of multi-spectral scanner systems. Hyperspectral sensors can help to overcome the traditional problems like the saturation problem in estimating quantity and the estimation of quality[2]. Hyperspectral sensors collect data as a set of images in which each image represents a narrow band wavelength range of the electromagnetic spectrum also known as a spectral band. These images are combined to form a three dimensional hyperspectral data cube for processing and analysis, where $\mathrm{x}$ and $\mathrm{y}$ represent the two spatial dimensions and $\mathrm{z}$ represents the spectral dimension of the scene [8]. There are multiple techniques to acquire the hyperspectral datasets and the selection of the technique is application 
specific [8]. Hyperspectral remote sensing technology is utilized in a wide array of applications. In addition to mapping distribution of materials, assessment of individual pixel is often useful for detecting unique objects in the scene. Scientific application areas include agriculture and forestry, ecology, atmosphere studies, geology and mineral exploration, marine, coastal zone management, inland waters and wetlands, urban development, snow and ice [3]. There are also numerous military applications in camouflage, littoral zone mapping, and landmine detection. Hyperspectral sensors have an advantage over multispectral sensors in their ability to identify and quantify molecular absorption. The higher spectral resolution of a hyperspectral imager is suitable for detection, identification and quantification of surface materials, as well as inferring biological and chemical processes [3]. For all of these applications, ground truth signatures collected in the field and indexed in spectral libraries are critical for many methods of analysis. Like other applications, hyperspectral remote sensing also has its own advantages and disadvantages. The objective of this paper is to review the technique in terms of data acquisition, processing, potential applications in multidisciplinary areas, advantages and disadvantages and future possibilities.

\section{HYPERSPECTRAL DATA AVAILABILITY AND AQUISITION}

Hyperspectral data is available from different types and levels of sensors like ground based, aircraft based and satellite based. The ground based sensors are Analytical Spectral Device Inc., Geophysical and Environmental Res. Corp and various airborne hyperspectral sensors are AIS (450-880 nm, 14 bands, 3 $\mathrm{nm}$ ) of Indian Space Research Organization, CASI (458-1000 nm, 96 bands, $6.8 \mathrm{~nm}$ ) and AVIRIS (380$2500 \mathrm{~nm}, 224$ bands, $10 \mathrm{~nm}$ ) of National Aeronautics and Space Administration. Hyperion onboard EO-1 satellite is the first space borne hyperspectral sensor, operating across the full solar reflected spectrum with nominal spectral coverage from 400-2500 $\mathrm{nm}$ [7]. There are four basic techniques for acquiring the three-dimensional $(\mathrm{x}, \mathrm{y}, \mathrm{z})$ dataset of a hyperspectral cube. Those techniques are spatial scanning, spectral scanning, non-scanning, spatio-spectral scanning. The choice of technique depends on the specific application, seeing that each technique has context-dependent advantages and disadvantages.

\section{APPLICATIONS OF HYPERSPECTRAL REMOTE SENSING IN VARIOUS DISCIPLINES}

Agriculture and Forestry:

Remote sensing has number of applications in the field of agriculture [10]. Remote sensing data acquired from sensors have been used for various applications such as crop forecasting, cropping system analysis, precision farming, horticulture, crop parameter retrieval, irrigation management, watershed development etc [12]. However, there are many limitations in the broadband data in discriminating similar crops, detecting various biotic and abiotic stresses and accurate retrieval of biochemical and biophysical parameters [8]. Hyperspectral remote sensing is very helpful to overcome some of these limitations and can improve the crop growth monitoring. The high spectral resolution reflectance spectrum in the region from 400-2500 $\mathrm{nm}$ is useful to identify large range of surface cover parameters that cannot be identified with broadband, low spectral resolution imaging system [7]. Therefore, hyperspectral remote sensing is very useful in crop discrimination, disease identification and stress identification within the crop. It is possible to compute narrow band indices using spectral band combinations related to biophysical parameters like Leaf Area Index (LAI) and biochemical crop parameters like chlorophyll content, water content and nitrogen content. Parameters like LAI, chlorophyll content, water content and nitrogen content indicate physiological state and stress conditions which affect crop yield and therefore it is important to establish mathematical relationship between various hyperspectral indices and crop parameters. [9].Various characteristics like species, LAI, biomass stress can be assessed effectively by hyperspectral vegetation indices because these indices reduce multispectral remote sensing data to single numbers. [12]. Hyperspectral vegetation indices are related to absorbed total solar and photosynthetically active radiation, canopy photosynthesis, stomatal conductance and land surface albedo. Moreover, by utilization of these indices errors associated with atmospheric effect, soil background effect and sensor viewing geometry can be eliminated [11]. Normalized Difference Vegetation Index (NDVI), Simple Ratio 
(SR), Soil Adjusted Vegetation Index (SAVI), Chlorophyll Absorption Ratio Index (CARI), Red Edge NDVI, Red Edge Chlorophyll Index is important vegetation indices [10]. Forestry is another field having multiple applications of hyperspectral remote sensing. Planning and management tasks associated with forest require accurate information about forest resources. The spatial distribution of trees in mixed species forest canopies is one of the most important information needed in the field of forestry for conservation and management [11]. Most remote sensing systems in the past decades rely on measured reflectance data in a few broad wavelength bands [11]. Conservation and monitoring of forest health is very essential for human lives in terms of social, economical and ecological aspects [11]. Hyperspectral remote sensing is a powerful tool utilized in many forest areas for forestry applications like forest planning management, forest mapping, forest inventory, forest area monitoring etc.

Geology and mineral exploration:

Geological mapping and mineral exploration techniques are tedious, expensive and time-consuming [3]. These traditional techniques involve extensive fieldwork, structural mapping, and study of landforms, petrography, mineralogy and geochemical analyses. These techniques need a strong laboratory database to discern slight variation in composition of ore grades [3]. Lithological discrimination and mineral potential mapping are possible with the multispectral sensors having bands in the Shortwave Infrared (SWIR) and Thermal Infra-Red (TIR) regions. Detailed understanding on precise mineral composition and relative abundance of constituents is not possible with multispectral data due to coarse bandwidth and poor spectral contiguity [3]. These limitations of multispectral remote sensing can be eliminated by hyperspectral remote sensing [2]. Hyperspectral remote sensing has many potential applications in the field of geology and mineral exploration from lithological mapping to exploration of economic minerals [3]. Hyperspectral remote sensing technology is an effective tool for geological applications like lithological mapping and exploration of industrial minerals, mapping of hydrothermal alteration zones and associated metal deposits and hydrocarbon exploration [3]. Hyperspectral remote sensing in the VNIR-SWIR region is widely used for lithologic mapping in various climatic and litho-tectonic terrane (granite, ophiolites84, peridotites and kimberlite) [3]. Mineral spectral features in the TIR region are mostly attributed to fundamental vibrations (bends and stretches) of $\mathrm{Si}-\mathrm{O}$ bonds. Thus, the TIR region spectrum is effectively utilized for the characterizing spectral features of many rock-forming minerals such as quartz, feldspar, amphibole, olivine, mica and pyroxene [3].

\section{Ecology:}

Ecological studies involve the investigation of organisms and their surrounding environment, including biotic and abiotic entities [9]. Mapping and monitoring biodiversity is very tedious due to the multifaceted nature of biodiversity [9]. By utilization of hyperspectral remote sensing data it is possible to collect ample spectral information across the spectrum generally [9]. Subtle differences between objects of interest can be estimated by accurate hyperspectral data collection and application. Hyperspectral data have been successfully utilized in recording information regarding critical plant parameters like leaf pigment, water content and chemical composition, discriminating tree species in landscapes, and fairly accurate identification between different species [9]. Validated spectral libraries are useful in land-cover classification, characterization and change detection. Accurate estimation of ecological processes, biodiversity monitoring of forest, grassland, vegetation, ecosystem monitoring, ecological succession and coastal zone environment monitoring is possible by incorporating hyperspectral remote sensing techniques [9]. Accurate classification can be applied for the discrimination of land cover in a complex natural ecosystem. Critical ecological parameters like canopy nutrient and moisture content, crown area, leaf-level drought responses, woody tissue and surface litter abundance, phenological patterns, and landcover transitions can be estimated by hyperspectral remote sensing [3]. Ecological research has many possible applications of hyperspectral remote sensing. 


\section{Coastal Zone Management:}

Due to development of industries, trade and commerce, tourism and resultant human population growth and migration, and deteriorating water quality the coastal zones are under increasing stress [13]. Coastal zones are important components of the global life system having very high biological productivity [8]. Coastal zones are recognized as a key ecosystem in conservation efforts of biodiversity [9]. Hyperspectral remote sensing can used for mapping, management, change detection and other aspects of ecological research. Growing population and coastal zone development needs advancement in technologies which enhances the mapping and monitoring of coastal zone management [8]. Application of airborne and field based hyperspectral sensors can provide ample data to carry out change detection, validation and spectral library generation [8]. Equally significant are the narrow and close spacing of the spectral bands [9]. These advanced sensors have a high potential to measure unique spectral responses of different ecological conditions like mapping and monitoring of coastal ecosystems and marine living resources, protection of shoreline, monitoring of coastal water quality, coastal hazards and climate change and coastal zone development [9].

\section{ADVANTAGES AND DISADVANTAGES OF HYPERSPECTRAL REMOTE SENSING}

In hyperspectral remote sensing entire spectrum is acquired at each point therefore needs no prior knowledge of the sample. Post processing of the datasets gives all the possible information regarding the sample. Hyperspectral remote sensing gives detailed information about spectral-spatial models for classification and segmentation. Hyperspectral remote sensing is cost effective and provides nondestructive sampling. Larger data storage capacity, fast computers, sensitive detectors are requirements to carry out hyperspectral imaging studies due to larger and multidimensional data cubes. Innovative techniques are required to process the hyperspectral data and new programming skills need to be developed. Different analytical techniques are yet to be developed for hyperspectral remote sensing.

\section{FUTURE DIRECTIONS}

Hyperspectral remote sensing generates hundreds of spectral bands at various spectral bands for the area of the earth. Effective computation and image processing can be extremely fruitful in many application domains. Further research in this technology is potential to lead towards better scenario in the field of remote sensing.

\section{REFERENCES}

[1] Ali A. Darvishsefat, Tobias W. Kellenberger, Kalaus I. Itten (2002) Application of Hyperspectral Data for Forest Stand Mapping. Symposium on Geospatial Theory, Processing and Applications, Ottawa, 2002.

[2] Boochs, F., Kupfer, G., Dockter, K., \& Kubauch, W. (1990). Shape of the red as vitality indicator for plants. International Journal of Remote Sensing, 11, 1741 - 1753.

[3] Clark, A.F. (1999). Spectroscopy of rocks and minerals and principles of spectroscopy. In: A.N. Rencz (Editor), Manual of Remote Sensing: Remote Sensing for the Earth Sciences. John Wiley and Sons, NewYork, pp. 3-58.

[4] Driss Haboudane, John R. Miller, Elizabeth Pattey, Pablo J. Zarco-Tejada, Ian B. Strachan (2004) Hyperspectral vegetation indices and novel algorithms for predicting green LAI of crop canopies: Modeling and validation in the context of precision agriculture Remote Sensing of Environment 90 (2004) $337-352$ 
[5] D. Ramakrishnan and Rishikesh Bharti (2015) Hyperspectral remote sensing and geological Applications. CURRENT SCIENCE, VOL. 108, NO. 5, 10 MARCH 2015

[6] José M. Bioucas-Dias, Antonio Plaza, Gustavo Camp s-Valls, Paul Scheunders, Nasser M. Nasrabadi, Jocelyn Ch anussot (2013) IEEE Geoscience and remote sensing magazine, June 2013

[7] Kumar, L., Schmidt, K.S., Dury, S., \& Skidmore, A.K. (2001). Imagingspectrometry and vegetation science. F. van der Meer \& S.M. de Jong (Editors), Imaging spectrometry. Kluwer Academic publishers, Dordrecht, The Netherlands, pp. 111-155.

[8] Kumar, L., Rietkerk, M., van Langevelde, F., van de Koppel, J., van Andel, J., Hearne, J., de Ridder, N., Stroosnijder, L., Skidmore, A.K., \& Prins, H.H.T. (2002). Relationship between vegetation growth rates at the onset of the wet season and soil type in the Sahelof Burkina Faso:

implications for resource utilisation at large scales. Ecological Modelling, 149, 143 - 152.

[9] Kai Wang, Steven E. Franklin, Xulin Guo, Marc Cattet (2010) Remote Sensing of Ecology, Biodiversity and Conservation: A Review from the Perspective of Remote Sensing Specialists. Sensors 2010, 10, 9647-9667; doi:10.3390/s101109647

[10] M Govender, K Chetty and H Bulcock (2006) A review of hyperspectral remote sensing and its application in vegetation and water resource studies. Water SA Vol. 33 No. 2 April 2007 (Online).

[11] Mantesh Gudimani, Shivraj Singh, Iyyanki V Muralikrishna, M V Raghunadh and V V Mani (2014) Application of hyperspectral remote sensing for land degradation and forest health monitoring using multispec. ISRS Proceeding Papers of Sort Interactive Session ISPRS TC VIII International Symposium on "Operational Remote Sensing Applications: Opportunities, Progress and Challenges", Hyderabad, India, December 9 - 12, 2014.

[12] Ray, S. S. and Dadhawal, V.K. (1995) Spectral Vegetation indices: Recent Advances. Signatures (Newaletter of ISRS-AC), 9 (1\&2): 5-9.

[13] Thenkabail, S.P., Enclona, E.A., Ashton, M., and Van Der Meer, B. 2004. Accuracy assessments of hyperspectral waveband performance for vegetation analysis applications. Remote Sensing of Environment. Vol. 91, No. 3-4, ppL 354-376 